\title{
Psychological Distress and Illness Perceptions in Thyroid Cancer Survivors: Does Age Matter?
}

\author{
Olga Husson, $\mathrm{PhD},{ }^{1,2}$ Hanneke Poort, $\mathrm{PhD},{ }^{3}$ Ursula M. Sansom-Daly, $\mathrm{PhD},{ }^{4,5}$ \\ Romana Netea-Maier, $\mathrm{PhD}{ }^{6}$ Thera Links, $\mathrm{PhD},{ }^{7}$ and Floortje Mols, $\mathrm{PhD}^{8,9}$
}

\begin{abstract}
Purpose: To examine the role of differentiated thyroid cancer (DTC) patients' age in illness perceptions and psychological distress, and the potential moderating role of age in the relationship between illness perceptions and psychological distress.

Methods: We used the Netherlands Cancer Registry to select all patients diagnosed with thyroid cancer between 1990 and $2008(n=568)$. Patients filled out the Hospital Anxiety and Depression Scale (HADS) and Brief Illness Perception Questionnaire. Levels of psychological distress and illness perceptions were compared between the different DTC survivor age groups (adolescents and young adults [AYAs; 18-39 years], middleaged adults [40-64 years], and older survivors [65-84 years]).

Results: Among 293 respondents with DTC, AYAs $(n=84)$ had more faith in the fact that their treatment can help them, and reported a stronger belief in understanding their illness compared to middle-aged $(n=172)$ and older adults $(n=37)$. No differences regarding age were seen on the other six illness perception subscales. AYAs did report significantly less distress (HADS caseness $=13.8 \%)$ compared to middle-aged $(28.7 \%)$ and older adults $(22.2 \%)$. Most illness perception subscales were associated with distress and the associations with age were mixed. AYAs and older patients who believed that their illness would continue for a long time reported more distress, but this association was not found for the middle-aged group.

Conclusion: Illness perceptions play a key role in the experience of distress years after diagnosis and this is related to age. AYA and older cancer patients may be particularly vulnerable to distress related to maladaptive cancer-related beliefs/perceptions.
\end{abstract}

Keywords: thyroid cancer, psychological distress, illness perceptions, age

\section{Introduction}

$\mathbf{T}$ HYROID CANCER IS one of the less frequent malignancies worldwide; the age-standardized Europe incidence rate is 6.3 per 100,000 per year (3.1 in men and 9.3 in women). ${ }^{1}$ However, it is the most common endocrine malignancy and incidence rates are increasing. ${ }^{2-4}$ Differentiated thyroid cancer (DTC) includes papillary or follicular thyroid cancer, and generally has a very good prognosis with 20 -year relative survival rates of up to $95 \%,{ }^{5}$ resulting in a high prevalence of survivors. The majority of DTC patients are diagnosed with limited disease. ${ }^{6}$ Treatment involves surgery, predominantly (near-) total thyroidectomy, often followed by radioactive iodine therapy, which results in a lifelong dependence on substitution therapy with levothyroxine. DTC persists or recurs in $\pm 20 \%$ of patients and distant metastases develop in $\pm 13 \%$ of patients, even decades after diagnosis. $^{7}$

\footnotetext{
${ }^{1}$ Institute of Cancer Research and Royal Marsden NHS Foundation Trust, London, United Kingdom.

${ }^{2}$ Department of Psychosocial Research, Division of Psychosocial Research \& Epidemiology, The Netherlands Cancer Institute, Amsterdam, The Netherlands.

${ }^{3}$ Department of Psychosocial Oncology and Palliative Care, Dana-Farber Cancer Institute, Boston, Massachusetts, USA.

${ }^{4}$ School of Women's and Children's Health, UNSW Sydney, Sydney, Australia.

${ }_{5}^{5}$ Behavioural Sciences Unit, Kids Cancer Centre, Sydney Children's Hospital, Randwick, Australia.

${ }^{6}$ Division of Endocrinology, Department of Internal Medicine, Radboud University Medical Center, Nijmegen, The Netherlands.

${ }^{7}$ Department of Endocrinology, University Medical Center Groningen, University of Groningen, Groningen, The Netherlands.

${ }^{8}$ Department of Research, Netherlands Comprehensive Cancer Organisation (IKNL), Utrecht, The Netherlands.

${ }^{9}$ Department of Medical and Clinical Psychology, CoRPS-Center of Research on Psychological and Somatic Disorders, Tilburg University, Tilburg, The Netherlands.
} 
While some studies found that DTC patients have a healthrelated quality of life (HRQoL) comparable to those in the general population, ${ }^{8,9}$ most recent studies report lower levels of physical and psychosocial functioning. Also, there are significantly more physical symptoms compared to an ageand sex-matched normative population. ${ }^{10,11}$ More specifically, DTC cancer patients report greater levels of anxiety and depression and lower levels of emotional functioning, and evidence suggests that their psychological distress persists throughout long-term survivorship. ${ }^{8,11-13}$

In general, a major determinant of psychological distress, next to the cancer itself, is the way patients perceive and respond to their illness. ${ }^{6}$ Research on these illness perceptions is guided by the self-regulation model. ${ }^{14}$ This model is based on the assumption that patients respond to symptoms and signs of illness by forming cognitive and emotional representations of the illness, which lead to coping responses. Various cancer studies show that threatening or negative illness perceptions predicted worse HRQoL and depression after treatment. ${ }^{15-21}$ There has been only one study in DTC patients showing high emotional concerns, which found that patients' emotional concerns were highly correlated with low perceived coherence and more severe consequences of the disease. ${ }^{6}$ However, this study did not examine the relationship between psychological distress and illness perceptions.

It is critical to understand how patient characteristics affect psychological distress and illness perceptions, and incorporate such factors into supportive care. For instance, different health issues can develop depending on age. For example, adolescents and young adults (AYAs) experience their disease during a highly complex developmental period and the peak time of onset of mental health disorders. ${ }^{22}$ Consequently, they report higher levels of psychological distress compared to older cancer patients. ${ }^{22}$ Middle-aged patients may have attained greater financial and social stability relative to their AYA counterparts; however, while also managing often the busiest periods of their careers, they can also be burdened with multiple caring responsibilities, including young children and older parents/relatives. ${ }^{23}$ Older patients also experience comorbid conditions alongside their cancer, as well as associated reductions in independence. ${ }^{24,25}$ However, it is not clear which age groups may be more psychologically "vulnerable" in the context of a DTC diagnosis. While middle-aged and older cancer patients have more life experience than AYAs, this could possibly influence their psychological distress and illness perceptions positively (e.g., through having a greater set of learned coping strategies and more social resources) or negatively (e.g., through having witnessed more peers or family members with poor health or cancer outcomes). Understanding the relative psychosocial vulnerability of different patient age groups will assist in best tailoring and targeting existing supportive care resources to the patient subgroups most in need.

Our aim is to examine the role of age in illness perceptions and psychological distress and the potential moderating role of age in the relationship between illness perceptions and psychological distress among DTC patients. Three specific research questions were posed:

(1) How do DTC survivors perceive their illness, and does this differ by age group?
(2) How distressed are DTC survivors across different age groups?

(3) Do illness perceptions impact upon DTC survivors' distress, and if so, does this differ by age group?

\section{Methods}

\section{Setting and population}

This study was based upon a population-based survey among thyroid cancer survivors registered within the Netherlands Cancer Registry (NCR), which compiles data of all individuals newly diagnosed with cancer. ${ }^{26}$ The NCR was used to select everyone diagnosed with thyroid cancer between 1990 and $2008(n=568)$ in the southern part of the Netherlands, an area with 2.3 million inhabitants. We excluded patients with cognitive impairment or those who, according to their treating physician, were deemed too ill at time of the study to fill out questionnaires $(n=31)$, those with unverifiable addresses $(n=90)$, and those who died before the start of the study according to the NCR, hospital records, and the Central Bureau for Genealogy $(n=6) .{ }^{26}$ One hospital refrained from participation in the study $(n=86)$. We sent invitations to participate to the remaining 355 thyroid cancer survivors. A flowchart of the data collection process has been previously published. ${ }^{11}$

\section{Data collection}

We collected data in November 2010 within PROFILES (Patient Reported Outcomes Following Initial treatment and Long-term Evaluation of Survivorship), a registry for the study of the physical and psychosocial impact of cancer and its treatment. ${ }^{27}$ PROFILES contains a large web-based component and is linked directly to clinical data from the NCR. Details of the data collection methods were previously described. ${ }^{11}$

Eligible patients were informed about the study by a letter from their (former) treating physician. ${ }^{26}$ Interested patients could provide informed consent and complete questionnaires online. Paper-and-pencil versions of the informed consent form and questionnaire were available upon request for those who preferred this. ${ }^{26}$ The certified Medical Ethics Committee of the Maxima Medical Centre, the Netherlands, approved this study.

\section{Study measures}

Sociodemographic and clinical characteristics. We attained sociodemographic and clinical characteristics (e.g., date of diagnosis, TNM stage, primary treatment, sex, and age) from the NCR. We used a self-report questionnaire to assess marital status, educational level, and current occupation. Comorbidity at time of survey was measured with the adapted Self-administered Comorbidity Questionnaire. ${ }^{28}$

Psychological distress. Since symptoms of cancer may overlap with somatic complaints of depression (e.g., feeling exhausted, change in appetite, and weight loss), we used the Hospital Anxiety and Depression scale (HADS) to assess self-reported depressive symptoms. ${ }^{29}$

The HADS consists of 14 items, seven items for depressive symptoms and seven items for anxiety. ${ }^{29}$ It assesses levels of 
symptoms in the last week. Items are answered on a fourpoint Likert scale and the total score for each scale ranges from 0 to 21 . HADS caseness was determined with a cutoff score $>11 .^{30}$

Illness perceptions. We assessed illness perceptions with the Brief Illness Perception Questionnaire (BIPQ), which was designed to assess cognitive and emotional representations of the illness. ${ }^{31}$ The BIPQ uses a single-item scale approach to assess perceptions on a continuous linear 0-10 point scale. ${ }^{31}$ Five of the items assess cognitive illness representations: (1) How much does your illness affect your life (consequences); (2) How long do you think your illness will continue (timeline); (3) How much control do you feel you have over your illness (personal control); (4) How much do you think your treatment can help your illness (treatment control); and, (5) How much do you experience symptoms from your illness (identity). ${ }^{31}$ Two items assess emotional

Table 1. Sociodemographic and Clinical Characteristics of Thyroid Cancer Survivors Stratified by Age Category at the Time of Questionnaire

\begin{tabular}{|c|c|c|c|c|}
\hline & AYAs $(\mathrm{n}=84)$ & Middle-aged adults $(\mathrm{n}=172)$ & Older adults $(\mathrm{n}=37)$ & $\mathrm{p}$ \\
\hline & \multicolumn{4}{|c|}{ Mean (SD) or n (\%) } \\
\hline Mean age at diagnosis & $29.1(5.0)$ & $49.1(8.0)$ & $72.5(4.5)$ & $<0.0001$ \\
\hline Mean time since diagnosis (years) & $11.2(5.4)$ & $9.5(5.3)$ & $6.4(4.4)$ & $<0.0001$ \\
\hline $\begin{array}{l}\text { Time since diagnosis (years) } \\
<5 \\
5-10 \\
>10\end{array}$ & $\begin{array}{l}15(17.9) \\
20(23.8) \\
49(58.3)\end{array}$ & $\begin{array}{l}45(26.2) \\
51(29.7) \\
76(44.2)\end{array}$ & $\begin{array}{r}18(48.6) \\
10(27.0) \\
9(24.3)\end{array}$ & 0.002 \\
\hline Mean age at time of survey & $40.8(7.6)$ & $59.1(9.3)$ & $79.4(5.0)$ & $<\mathbf{0 . 0 0 0 1}$ \\
\hline $\begin{array}{l}\text { Gender } \\
\text { Male } \\
\text { Female }\end{array}$ & $\begin{array}{l}15(17.9) \\
69(82.1)\end{array}$ & $\begin{array}{r}43(25.0) \\
129(75.0)\end{array}$ & $\begin{array}{l}12(32.4) \\
25(67.6)\end{array}$ & 0.194 \\
\hline Marital status & & & & \\
\hline $\begin{array}{l}\text { Married/cohabiting } \\
\text { Divorced/widowed/never married }\end{array}$ & $\begin{array}{l}72(85.7) \\
12(14.3)\end{array}$ & $\begin{array}{r}138(80.2) \\
34(19.8)\end{array}$ & $\begin{array}{l}18(48.6) \\
19(51.4)\end{array}$ & $<0.0001$ \\
\hline $\begin{array}{l}\text { Educational level }^{\mathrm{a}} \\
\text { Lower education } \\
\text { Medium education } \\
\text { Higher education }\end{array}$ & $\begin{aligned} & 3(3.6) \\
52 & (61.9) \\
29 & (34.5)\end{aligned}$ & $\begin{array}{r}17(9.9) \\
112(65.5) \\
42(24.6)\end{array}$ & $\begin{array}{r}11(29.7) \\
20(54.1) \\
6(16.2)\end{array}$ & $<\mathbf{0 . 0 0 0 1}$ \\
\hline $\begin{array}{l}\text { Current occupation }^{\mathrm{b}} \\
\text { Employed } \\
\text { Unemployed }\end{array}$ & $\begin{array}{l}67(82.7) \\
14(17.3)\end{array}$ & $\begin{array}{l}82(48.2) \\
88(51.8)\end{array}$ & $\begin{array}{c}0(0) \\
37(100)\end{array}$ & $<\mathbf{0 . 0 0 0 1}$ \\
\hline $\begin{array}{l}\text { Number of comorbid conditions (mean) } \\
\text { Comorbidity }\end{array}$ & $1.5(1.6)$ & $2.2(1.8)$ & $2.6(2.4)$ & $\begin{array}{l}<0.001 \\
<0.001\end{array}$ \\
\hline $\begin{array}{l}0 \\
1 \\
\geq 2\end{array}$ & $\begin{array}{l}24(28.6) \\
29(34.5) \\
31(36.9)\end{array}$ & $\begin{array}{r}34(19.8) \\
28(16.3) \\
110(64.0)\end{array}$ & $\begin{array}{r}9(24.3) \\
7(18.9) \\
21(56.8)\end{array}$ & \\
\hline $\begin{array}{l}\text { Type of thyroid cancer } \\
\text { Papillary } \\
\text { Follicular (including Hürthle cell) }\end{array}$ & $\begin{array}{l}68(81.0) \\
16(19.0)\end{array}$ & $\begin{array}{r}128(74.9) \\
43(25.1)\end{array}$ & $\begin{array}{l}22(61.1) \\
14(38.9)\end{array}$ & 0.071 \\
\hline $\begin{array}{l}\text { TNM stage } \\
\text { I } \\
\text { II } \\
\text { III } \\
\text { IV }\end{array}$ & $\begin{array}{l}84(100) \\
0(0) \\
0(0) \\
0(0)\end{array}$ & $\begin{array}{l}80(47.9) \\
43(25.7) \\
33(19.8) \\
11(6.6)\end{array}$ & $\begin{aligned} 5 & (14.3) \\
10 & (28.6) \\
11 & (31.4) \\
9 & (25.7)\end{aligned}$ & $<0.0001$ \\
\hline $\begin{array}{l}\text { Primary treatment } \\
\text { Surgery alone } \\
\text { Surgery }+{ }^{131} \text { I ablation } \\
\text { Surgery }+ \text { radiotherapy } \\
\text { Other }\end{array}$ & $\begin{array}{l}22(26.2) \\
61(72.6) \\
1(1.2) \\
0(0)\end{array}$ & $\begin{aligned} 42 & (24.4) \\
121 & (70.3) \\
7 & (4.1) \\
2 & (1.2)\end{aligned}$ & $\begin{aligned} 6 & (16.2) \\
30 & (81.1) \\
1 & (2.7) \\
0 & (0)\end{aligned}$ & 0.602 \\
\hline
\end{tabular}

AYAs (18-39 years), middle-aged adults (40-64 years), and older adults (65-84 years): age at time of diagnosis.

Some variables exceed $100 \%$ due to rounding off and some variables do not add up to $100 \%$ because of missing data.

Bold indicates the scales are significant.

${ }^{a}$ Education: Low (no education or primary school); Medium (lower general secondary education or vocational training); and High (high vocational training or university).

${ }^{\mathrm{b}}$ Current occupation: Unemployed also includes individuals who were students, retired, incapacitated due to illness/disability, or those in full-time caring roles such as stay-at-home parents.

Occupational status missing for 1 AYA and 2 middle-aged adults; educational level missing for 1 middle-aged adult; TNM stage missing for 5 middle-aged adults and 2 older adults; and type of thyroid cancer missing for 1 middle-aged adult and 1 older TCS.

AYAs, adolescents and young adults; SD, standard deviation; TCS, thyroid cancer survivor. 
representations: (6) How concerned are you about your illness (concern); and (7) How much does your illness affect you emotionally (emotional representation). ${ }^{31}$ One item assesses illness comprehensibility: (8) How well do you understand your illness (coherence). ${ }^{31}$

\section{Statistical analyses}

We compared differences in clinical and sociodemographic characteristics between respondents, nonrespondents, and patients with unverifiable addresses; and between AYAs (18-39 years), middle-aged adults (40-64 years), and older (65-84 years) DTC survivors using chi-square statistics for categorical variables and $t$-tests or analysis of variance (ANOVA) for continuous variables.

ANOVA was used to compare levels of psychological distress and illness perceptions between the different DTC survivor age groups.

Eight multiple linear regression analyses were conducted with continuous illness perceptions as outcomes. Each regression equation consisted of age group (mean centered), HADS total score (mean -centered), and their interaction term. ${ }^{32}$ Interactions are visualized using Hayes' PROCESS method. ${ }^{33}$

All these exploratory tests were two sided and considered statistically significant if $p<0.05$, given the exploratory nature of our study. Analyses were performed using IBM SPSS version 22.0 (Statistical Package for Social Sciences, Chicago, IL).

\section{Results}

\section{Sociodemographic and clinical characteristics}

As previously reported, the response rate was high $(86 \%)$ since 306 out of 355 survivors returned their completed questionnaire. ${ }^{11} \mathrm{~A}$ comparison among respondents, nonrespondents, and those with unverifiable addresses showed that those with unverifiable addresses were significantly younger than nonrespondents and respondents. ${ }^{11}$ No difference was seen with respect to sex, type of thyroid cancer, TNM classification, or primary treatment between these groups.

For this study, only patients with DTC were included $(n=293)$. We categorized them into three groups according to age at diagnosis; AYAs (18-39 years; $n=84)$, middle-aged adults $(40-64$ years; $n=172)$, and older survivors $(65-84$ years; $n=37)$. Patients in the three age groups differed significantly regarding time since diagnosis, marital status, educational level, current occupation, the number of comorbid conditions, and TNM classification (all $p$ values $<0.01$; Table 1). ${ }^{34}$ No difference was found between the three age groups with respect to gender, type of cancer (e.g., papillary and follicular), or primary treatment.

\section{Research question 1: how do DTC survivors perceive their illness, and does this differ by age group?}

We found significant differences between AYAs and middle-aged and older adults on two of the eight BIPQ subscales, "Treatment control"' and "Coherence." AYAs reported a greater sense of control and a better understanding of their illness (Table 2).

\section{Research question 2: how distressed are DTC survivors across different age groups?}

No difference was found between age groups regarding the continuous HADS score, but the proportion of HADS caseness differed significantly between AYAs (13.8\%) and middle-aged (28.7\%) and older adults (22.2\%; Table 2).

\section{Research question 3: do illness perceptions impact DTC survivors' distress, and if so, does this differ by age group?}

Across all age groups, individuals with more negative illness perceptions on the BIPQ subscales "Consequences,"

Table 2. Differences in Illness Perceptions and Psychological Distress Scores According to Age Categories

\begin{tabular}{|c|c|c|c|c|c|c|c|}
\hline & \multicolumn{2}{|r|}{ AYAs } & \multicolumn{2}{|c|}{ Middle-aged adults } & \multicolumn{2}{|c|}{ Older adults } & \multirow{2}{*}{$\frac{A N O V A}{\mathrm{p}}$} \\
\hline & $\mathrm{n}$ & Mean $(S D)$ & $\mathrm{n}$ & Mean $(S D)$ & $\mathrm{n}$ & Mean $(S D)$ & \\
\hline \multicolumn{8}{|l|}{$\mathrm{BIPQ}^{\mathrm{a}}$} \\
\hline Consequences & 79 & $3.9(2.7)$ & 161 & $4.0(2.5)$ & 34 & $3.2(2.3)$ & 0.26 \\
\hline Timeline & 79 & $6.5(3.8)$ & 158 & $7.2(3.7)$ & 35 & $5.6(3.9)$ & 0.06 \\
\hline Personal control & 79 & $5.8(3.0)$ & 160 & $5.7(3.2)$ & 33 & $4.8(3.2)$ & 0.29 \\
\hline Treatment control & 79 & $8.0(2.2)$ & 159 & $7.7(2.7)$ & 34 & $6.4(3.0)$ & 0.02 \\
\hline Identity & 79 & $4.0(2.7)$ & 162 & $4.3(2.9)$ & 33 & $3.2(2.2)$ & 0.16 \\
\hline Illness concern & 79 & $3.8(2.3)$ & 162 & $3.8(2.6)$ & 35 & $4.3(3.1)$ & 0.49 \\
\hline Coherence & 79 & $8.1(2.2)$ & 161 & $7.5(2.6)$ & 33 & $6.2(2.9)$ & 0.01 \\
\hline Emotional representation & 80 & $3.7(2.7)$ & 160 & $3.4(2.5)$ & 35 & $2.8(2.4)$ & 0.19 \\
\hline \multicolumn{8}{|l|}{ HADS $^{\mathrm{b}}$} \\
\hline HADS total & 80 & $7.0(5.8)$ & 159 & $8.5(6.5)$ & 28 & $9.0(5.7)$ & 0.14 \\
\hline HADS caseness & 80 & $11 / 80(13.8 \%)$ & 159 & $49 / 159(28.7 \%)$ & 28 & $8 / 28(22.2 \%)$ & 0.02 \\
\hline
\end{tabular}

Bold indicates the scales are significant.

${ }^{a}$ BIPQ; perceptions represent a continuous linear 0-10 point scale. Consequences: How much does your illness affect your life; Timeline: How long do you think your illness will continue; Personal control: How much control do you feel you have over your illness; Treatment control: How much do you think your treatment can help your illness; Identity: How much do you experience symptoms from your illness; Concern: How concerned are you about your illness; Emotional representation: How much does your illness affect you emotionally; Coherence: How well do you understand your illness.

${ }^{\mathrm{b}}$ HADS; A higher score means more psychological distress. HADS caseness was determined as a cutoff score $>11 .{ }^{30}$

ANOVA, analysis of variance; BIPQ, Brief Illness Perception Questionnaire; HADS, Hospital Anxiety and Depression Scale. 


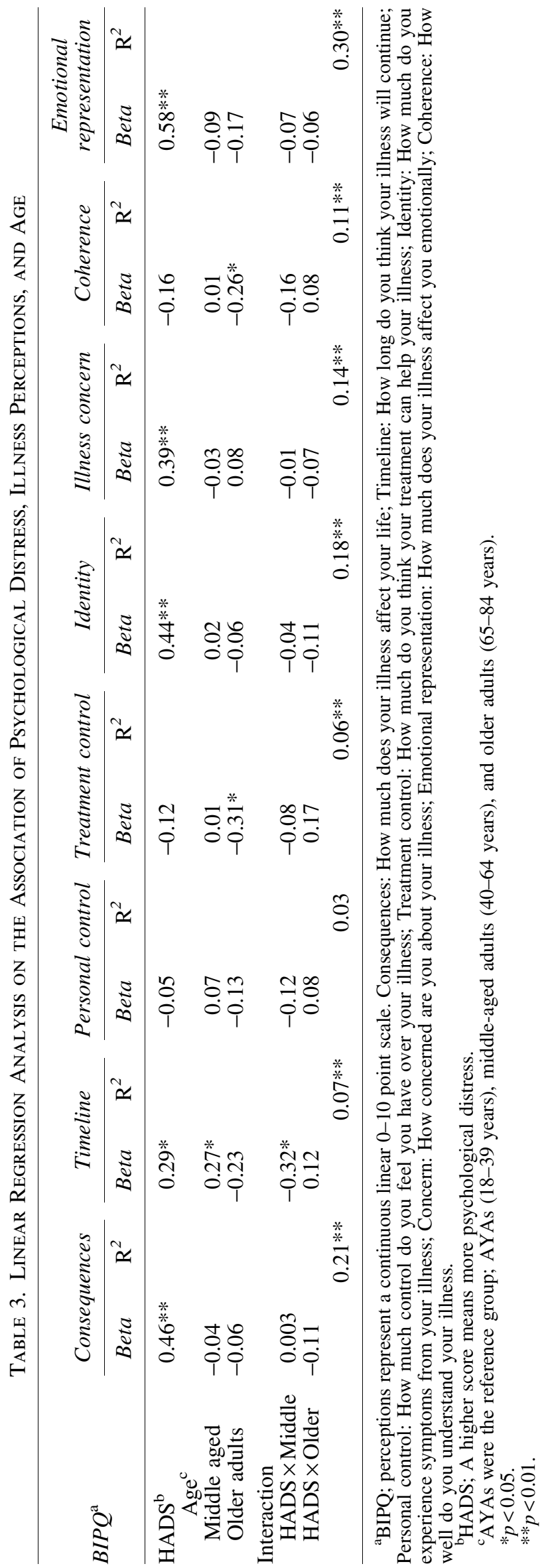

"Timeline," “Identity," "Illness concern," and "Emotional representation" also showed significantly greater psychological distress (Table 3). Several significant effects of age also emerged. Relative to their middle-aged peers, AYAs showed less negative perceptions of their illness continuing into the future (BIPQ subscale "Timeline"). Furthermore, "Treatment control" and "Coherence" were negatively associated with age, such that relative to older cancer patients, AYAs demonstrated greater confidence in their control over their treatment, and their understanding of it. In addition, only the BIPQ subscale "Timeline" was negatively associated with the interaction term between distress and middle-aged status. No effect was seen for the interaction term between psychological distress and the older group. Figure 1 depicts regression plots for age and psychological distress per BIPQ subscale. This graph indicates that perceptions of disease "Timeline" had a differential association with distress according to survivors' age; both AYA-aged and older individuals who perceived that their illness would continue further into the future showed significantly greater distress (Total HADS score). By contrast, this was not the case for the middle-aged group.

\section{Discussion}

Our aim was to study the role of age regarding illness perceptions and psychological distress among DTC patients and to investigate the potential moderating role of age in the relationship between illness perceptions and psychological distress. Our results showed that those who were AYAs at diagnosis reported more faith in the fact that their treatment could help them compared to middle-aged and older adults. It might be the case that AYAs are more flexible and resilient compared to middle-aged and older adults, whose older age may also translate to an accumulation of other life stressors/stressful life experiences that may impact them psychologically. In addition, AYAs' lesser life experiences may also have meant that they had fewer personal experiences with cancer before their own, including negative experiences where peers or family members may have died from their disease. ${ }^{22}$ This may have meant that AYAs were able to focus more on the positive messages around curative treatment conveyed to them by their treating health professionals. Studies have also shown that AYAs report having a lot of respect for, and trust in their treating team and eschew alternative, peripheral sources of information such as friends or “Doctor Google,"' which may contain more cancer-related "horror stories." 35 It is also possible that health professionals genuinely communicate more positive/optimistic messages to patients who are AYA aged, relative to their older counterparts, given their youth and often relatively good health/physical fitness aside from their cancer diagnosis. Future studies should explore these different explanations.

Those receiving the diagnosis at AYA age reported a stronger belief in understanding their illness compared to middle-aged and older adults. It is possible that information provision to AYAs is more. It is also possible that AYAs may be less influenced by perceptions of power imbalances or hierarchies in the health care system, and consequently may be able to more assertively ask for information when needed. As a group, AYAs may also have varying levels of health literacy, with younger and less educated AYAs likely to have 


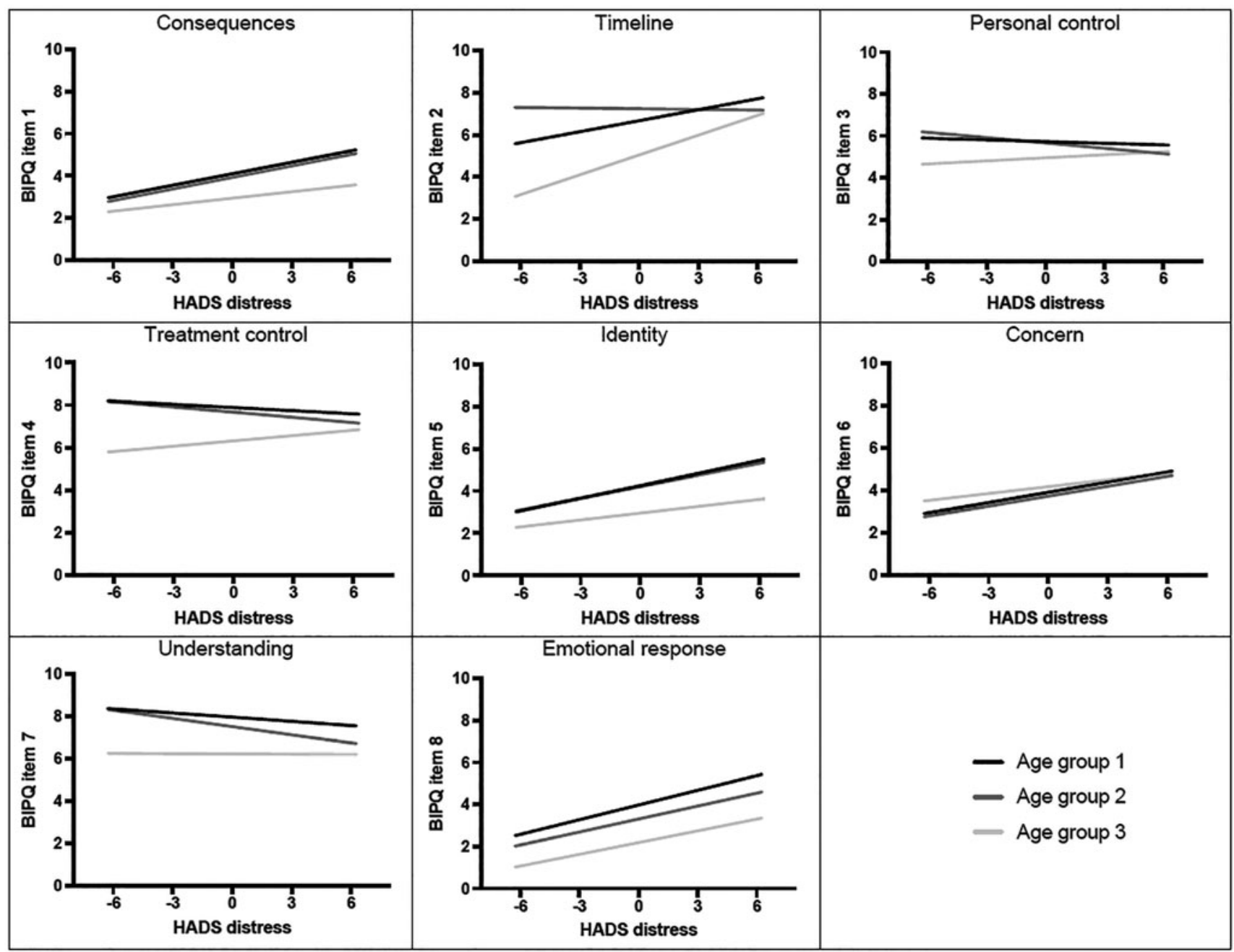

FIG. 1. Regression plots for age and psychological distress per BIPQ subscale. Age group 1: AYAs (18-39 years), Age group 2: middle-aged adults (40-64 years), and Age group 3: older adults (65-84 years). Illness perceptions were assessed with the BIPQ on a continuous linear 0-10 point scale. Question (1) How much does your illness affect your life; (2) Timeline: How long do you think your illness will continue; (3) Personal control: How much control do you feel you have over your illness; (4) Treatment control: How much do you think your treatment can help your illness; (5) Identity: How much do you experience symptoms from your illness; (6) Concern: How concerned are you about your illness; (7) Emotional representation: How much does your illness affect you emotionally; (8) Coherence: How well do you understand your illness. AYAs, adolescents and young adults; BIPQ, Brief Illness Perception Questionnaire.

underdeveloped health literacy skills. ${ }^{35,36}$ Therefore, and due to their stage of development, AYAs may also still defer to significant others (e.g., parents and partners) to share the responsibility of information gathering, processing, and retention with and for them. ${ }^{35}$ This may make the process of information provision less fraught and anxiety-provoking from AYAs' perspectives, and may also mean that as a group, AYAs selectively (and perhaps pragmatically) attend to and retain fewer key pieces of treatment-related information. If such social processes make the experience of information exchange easier for AYAs, this could also contribute to their sense of the process having been more helpful and comprehensive.

Results showed that AYAs (13.8\%) were less likely to report clinically relevant levels of psychological distress compared to middle-aged (28.7\%) and older adults $(22.2 \%)$. This contrasts to previous research. For example, a review, in- cluding 34 studies among cancer patients, concluded that, regardless of the specific age ranges used, AYAs are a distinct group with an elevated risk of distress, depression, and anxiety compared to older age groups. ${ }^{37}$ Differences between our DTC study and other cancer populations can possibly be explained by the relatively good prognosis of DTC. Our study also focused on long-term survivors. While distress can persist into survivorship, as time since diagnosis and treatment completion increases, so do the opportunities to psychologically process the experience and manage the cancer-related distress. ${ }^{22}$ Consequently, the distress seen in our study may have been driven by other noncancer-related factors.

As expected, higher scores on the following illness perception subscales (experiencing more consequences of illness, a stronger sense of continued duration of illness, experiencing more symptoms of the illness, being more concerned about the illness, and being more emotionally 
affected by the illness) were associated with more psychological distress in all age groups, which is in line with other studies. $^{38-40}$ This information can be used to inform therapeutic approaches to working with cancer-related distress.

Higher scores on two illness perception subscales (i.e., thinking your treatment can help your illness more and understanding your illness better) were associated with younger age, while higher scores on a third one (a stronger sense of continued duration of illness) was associated with higher age. AYAs experience their cancer amidst a challenging period of physical, emotional, cognitive and social development and changing economic demands. Being diagnosed with cancer can strongly interfere with the accomplishment of normal developmental milestones. ${ }^{41}$ On the other hand, AYAs may be more selective and/or pragmatic in attending only to key pieces of information, or may be more assertive to ask their health care provider for the information they desire. ${ }^{35} \mathrm{Re}-$ lative to their older counterparts, AYA cancer survivors need to attend long-term follow-up care for the longest time to undergo surveillance for recurrent and secondary cancers, as well as additional medical late effects. Understanding how these beliefs about the duration of their illness and treatment can be leveraged to maximize their engagement in long-term medical care is critical.

One unexpected finding was the fact that both AYAs and older survivors reported significantly more distress if they believed that their illness would continue for a long time, while this effect was not found for the middle-aged group. Future research should explore whether these perceptions around disease timelines impact age groups differently and why. Recent research has shown that relative to healthy controls, AYA cancer survivors show difficulties in effectively imagining their future and that this was associated with higher distress. ${ }^{42}$ Perceiving cancer as ongoing is likely to further challenge AYAs' capacities to imagine their futures-a process critical to their transition to wellfunctioning adulthood and a life of healthy survivorship.

Our study is (one of) the first to comprehensively examine the links between distress and illness perceptions among DTC survivors, with a specific focus on age. Although valuable, this study has limitations. We included patients diagnosed between 2 and 20 years ago, limiting the generalizability of our results to newly diagnosed patients. Furthermore, since this is a cross-sectional study, we could not determine whether causal associations exists between illness perception and distress. Also, the older adult age group had different sociodemographic and clinical characteristics at the time of survey compared to the other two age groups. Despite these differences, the differences between age group were small, which is an important finding. Finally, patients were categorized according to age at diagnosis, but they were between 2 and 20 years older at time of the questionnaire, which might be the reason that we did not find more differences between these groups regarding illness perception and distress.

Results of two other PROFILES studies showed that illness perceptions may be associated with lower HRQoL and survival. ${ }^{20,43}$ DTC patients' perceptions of their disease are thus important as they can influence health outcomes. A recent study on this same dataset showed that greater information support was associated with less threatening illness perceptions, and less threatening illness perceptions were in turn associated with less distress. ${ }^{44}$ Therefore, providing the right amount of information at the right time to survivors is important, and new studies on this topic are necessary. Research focused on understanding the longitudinal trajectory of survivors' illness perceptions and distress will be especially important among DTC survivors, as they visit their physician regularly for years after diagnosis and primary treatment to receive thyroid hormone replacement therapy. These regular contacts and continued treatment can be a constant reminder of DTC and can influence patients' illness perceptions and distress. In term, this could lead to dropout of follow-up care, while follow-up appointments could be an opportunity for screening on distress and maladaptive illness perceptions.

In conclusion, illness perceptions play a key role in the experience of distress years after diagnosis and this is related to patients' age. Therefore, health care professionals need to identify and address maladaptive illness perceptions and high levels of distress and refer patients to appropriate care to decrease psychological distress, and thereby improve survivorship.

\section{Acknowledgments}

We would like to thank all patients and their doctors from the following hospitals for their participation in this study: Bernhoven Hospital, Veghel and Oss; Catharina Hospital, Eindhoven; Elkerliek Hospital, Helmond and Deurne; Jeroen Bosch Hospital, Den Bosch; Maxima Medical Centre, Eindhoven and Veldhoven; Sint Anna Hospital, Geldrop; St. Elisabeth Hospital, Tilburg; Twee Steden Hospital, Tilburg; and VieCuri Hospital, Venlo and Venray.

\section{Author Disclosure Statement}

No competing financial interests exist.

\section{Funding Information}

The data collection of this study was funded by the Netherlands Comprehensive Cancer Organisation and a Medium Investment Grant from the Netherlands Organisation for Scientific Research (NWO no. 480-08-009). Dr. O.H. is supported by a Social Psychology Fellowship from the Dutch Cancer Society (no. KUN2015-7527). Dr. U.M.S.-D. is supported by Early Career Fellowships from the Cancer Institute New South Wales (ID: 14/ECF/1-11) and the National Health and Medical Research Council (NHMRC) Australia (ID: APP1111800). These funding agencies had no further role in study design; in the collection, analysis, and interpretation of data; in the writing of the article; and in the decision to submit the article for publication.

\section{References}

1. Ferlay J, Steliarova-Foucher E, Lortet-Tieulent J, et al. Cancer incidence and mortality patterns in Europe: estimates for 40 countries in 2012. Eur J Cancer. 2013;49(6): 1374-403.

2. Amphlett B, Lawson Z, Abdulrahman GO, Jr., et al. Recent trends in the incidence, geographical distribution, and survival from thyroid cancer in Wales, 1985-2010. Thyroid. 2013;23(11):1470-8. 
3. Carlberg M, Hedendahl L, Ahonen M, et al. Increasing incidence of thyroid cancer in the Nordic countries with main focus on Swedish data. BMC Cancer. 2016;16:426.

4. Morris LG, Tuttle RM, Davies L. Changing trends in the incidence of thyroid cancer in the United States. JAMA Otolaryngol Head Neck Surg. 2016;142(7):709-11.

5. Brenner H. Long-term survival rates of cancer patients achieved by the end of the 20th century: a period analysis. Lancet. 2002;360(9340):1131-5.

6. Hirsch D, Ginat M, Levy $S$, et al. Illness perception in patients with differentiated epithelial cell thyroid cancer. Thyroid. 2009;19(5):459-65.

7. Schlumberger MJ. Diagnostic follow-up of welldifferentiated thyroid carcinoma: historical perspective and current status. J Endocrinol Invest. 1999;22(11 Suppl):3-7.

8. Crevenna R, Zettinig G, Keilani M, et al. Quality of life in patients with non-metastatic differentiated thyroid cancer under thyroxine supplementation therapy. Support Care Cancer. 2003;11(9):597-603.

9. Schroeder PR, Haugen BR, Pacini F, et al. A comparison of short-term changes in health-related quality of life in thyroid carcinoma patients undergoing diagnostic evaluation with recombinant human thyrotropin compared with thyroid hormone withdrawal. J Clin Endocrinol Metab. 2006; 91(3):878-84.

10. Singer S, Lincke T, Gamper E, et al. Quality of life in patients with thyroid cancer compared with the general population. Thyroid. 2012;22(2):117-24.

11. Husson O, Haak HR, Buffart LM, et al. Health-related quality of life and disease specific symptoms in long-term thyroid cancer survivors: a study from the population-based PROFILES registry. Acta Oncol. 2013;52(2):249-58.

12. Hoftijzer HC, Heemstra KA, Corssmit EP, et al. Quality of life in cured patients with differentiated thyroid carcinoma. J Clin Endocrinol Metab. 2008;93(1):200-3.

13. Tagay S, Herpertz S, Langkafel M, et al. Health-related quality of life, depression and anxiety in thyroid cancer patients. Qual Life Res. 2006;15(4):695-703.

14. Leventhal H, Nerenz D, Steele D. Illness representations and coping with health threats. In: Baum A, Taylor S, Singer J (Eds). Handbook of psychology and health. IV. Hillside, NJ: Erlbaum; 1984; pp. 219-52.

15. Ashley L, Marti J, Jones H, et al. Illness perceptions within 6 months of cancer diagnosis are an independent prospective predictor of health-related quality of life 15 months post-diagnosis. Psychooncology. 2015;24(11):1463-70.

16. Hopman P, Rijken M. Illness perceptions of cancer patients: relationships with illness characteristics and coping. Psychooncology. 2015;24(1):11-8.

17. Keeling M, Bambrough J, Simpson J. Depression, anxiety and positive affect in people diagnosed with low-grade tumours: the role of illness perceptions. Psychooncology. 2013;22(6):1421-7.

18. Rozema H, Vollink T, Lechner L. The role of illness representations in coping and health of patients treated for breast cancer. Psychooncology. 2009;18(8):849-57.

19. Scharloo M, Baatenburg de Jong RJ, Langeveld TP, et al. Quality of life and illness perceptions in patients with recently diagnosed head and neck cancer. Head Neck. 2005; 27(10):857-63.

20. Thong MS, Kaptein AA, Vissers PA, et al. Illness perceptions are associated with mortality among 1552 colorectal cancer survivors: a study from the population-based PROFILES registry. J Cancer Surviv. 2016;10(5):898-905.
21. Traeger L, Penedo FJ, Gonzalez JS, et al. Illness perceptions and emotional well-being in men treated for localized prostate cancer. J Psychosom Res. 2009;67(5):389-97.

22. Sansom-Daly UM, Wakefield CE. Distress and adjustment among adolescents and young adults with cancer: an empirical and conceptual review. Transl Pediatr. 2013;2(4):167-97.

23. Chassin L, Macy JT, Seo DC, et al. The association between membership in the sandwich generation and health behaviors: a longitudinal study. J Appl Dev Psychol. 2010; 31(1):38-46.

24. Kaul S, Avila JC, Mutambudzi M, et al. Mental distress and health care use among survivors of adolescent and young adult cancer: a cross-sectional analysis of the National Health Interview Survey. Cancer. 2017;123(5):869-78.

25. Geue K, Brahler E, Faller H, et al. Prevalence of mental disorders and psychosocial distress in German adolescent and young adult cancer patients (AYA). Psychooncology. 2018;27(7):1802-9.

26. Mols F, Thong M, Denollet J, et al. Are illness perceptions, beliefs about medicines and Type D personality associated with medication adherence among thyroid cancer survivors? A study from the population-based PROFILES registry. Psychol Health. 2020;35(2):128-143.

27. van de Poll-Franse LV, Horevoorts N, Eenbergen MV, et al. The Patient Reported Outcomes Following Initial treatment and Long term Evaluation of Survivorship registry: scope, rationale and design of an infrastructure for the study of physical and psychosocial outcomes in cancer survivorship cohorts. Eur J Cancer. 2011;47(14):2188-94.

28. Sangha O, Stucki G, Liang MH, et al. The SelfAdministered Comorbidity Questionnaire: a new method to assess comorbidity for clinical and health services research. Arthritis Rheum. 2003;49(2):156-63.

29. Zigmond AS, Snaith RP. The hospital anxiety and depression scale. Acta Psychiatr Scand. 1983;67(6):361-70.

30. Vodermaier A, Millman RD. Accuracy of the Hospital Anxiety and Depression Scale as a screening tool in cancer patients: a systematic review and meta-analysis. Support Care Cancer. 2011;19(12):1899-908.

31. Broadbent E, Petrie KJ, Main J, Weinman J. The brief illness perception questionnaire. J Psychosom Res. 2006; 60(6):631-7.

32. Aiken LS, West SG. Multiple regression: testing and interpreting interactions. Thousand Oaks, CA: Sage Publications, Inc.; 1991.

33. Hayes AF. Introduction to mediation, moderation, and conditional process analysis. A regression-based approach. New York: Giulford Press; 2017.

34. Mols F, Schoormans D, Smit JWA, et al. Age-related differences in health-related quality of life among thyroid cancer survivors compared with a normative sample: results from the PROFILES registry. Head Neck. 2018; 40(10):2235-45.

35. Lin M, Sansom-Daly UM, Wakefield CE, et al. Health literacy in adolescents and young adults: perspectives from Australian cancer survivors. J Adolesc Young Adult Oncol. 2017;6(1):150-8.

36. Sansom-Daly UM, Lin M, Robertson EG, et al. Health literacy in adolescents and young adults: an updated review. J Adolesc Young Adult Oncol. 2016;5(2):106-18.

37. Lang MJ, David V, Giese-Davis J. The age conundrum: a scoping review of younger age or adolescent and young adult as a risk factor for clinical distress, depression, or anxiety in cancer. J Adolesc Young Adult Oncol. 2015;4(4):157-73. 
38. Kus T, Aktas G, Ekici $\mathrm{H}$, et al. Illness perception is a strong parameter on anxiety and depression scores in early-stage breast cancer survivors: a single-center crosssectional study of Turkish patients. Support Care Cancer. 2017;25(11):3347-55.

39. Zhang N, Fielding R, Soong I, et al. Illness perceptions as predictors of psychological distress among head and neck cancer survivors: a longitudinal study. Head Neck. 2018; 40(11):2362-71.

40. Dempster M, McCorry NK, Brennan E, et al. Illness perceptions among carer-survivor dyads are related to psychological distress among Oesophageal cancer survivors. J Psychosom Res. 2011;70(5):432-9.

41. Zebrack BJ. Psychological, social, and behavioral issues for young adults with cancer. Cancer. 2011;117(10 Suppl): 2289-94.

42. Sansom-Daly UM, Wakefield CE, Robertson EG, et al. Adolescent and young adult cancer survivors' memory and future thinking processes place them at risk for poor mental health. Psychooncology. 2018;27(12):2709-16.
43. de Rooij BH, Thong MSY, van Roij J, et al. Optimistic, realistic, and pessimistic illness perceptions; quality of life; and survival among 2457 cancer survivors: the populationbased PROFILES registry. Cancer. 2018;124(17):3609-17.

44. Wiener $\mathrm{CH}$, Cassisi JE, Paulson D, et al. Information support, illness perceptions, and distress in survivors of differentiated thyroid cancer. J Health Psychol. 2019;24(9): 1201-9.

Address correspondence to:

Floortje Mols, PhD

Department of Medical and Clinical Psychology CoRPS-Center of Research on Psychological and Somatic Disorders

Tilburg University

PO Box 90153

Tilburg 5000 LE

The Netherlands

Email: f.mols@tilburguniversity.edu 